\title{
Kultwagen mit Figuren aus Keramik im Osthallstattkreis?
}

\author{
Ceramic Cult Wagons with Figures in the Eastern Hallstatt Zone
}

\section{Alexandrine Eibner}

\begin{abstract}
The bronze cult wagon from Srettweg exemplifies in the eastern Hallstatt zone a vessel-bearing wagon as it is otherwise only known from North and Central Italy. Since bronze vessels are almost completely absent in graves of the Kalenderberg Culture, we can, in my opinion, suppose that they were replaced by ceramics. This assumption can be supported by two ceramic assemblages from richly furnished graves of the Kalenderberg Culture (Langenlebarn and Gemeinlebarn). This is probably why these cult wagons - like that one from Strettweg (A) with applied figures - were imitated in clay, or mounted on a base from organic material, for example glued on a wooden wagon. In this pictorial material we can probably recognise the portrayal of ritual acts, processions or myths, which, in my opinion, can be also found in the scenes of the Situla Art as well as on the cistae from Kröllkogel of Kleinklein (A), and in figural compositions of the Kalenderberg Culture - but they are always made using different artistic means of expression.
\end{abstract}

\section{Keywords}

East Hallstatt Culture, ceramic figures, cult wagons, processions

\begin{abstract}
Abstrakt
Im bronzenen Kultwagen von Strettweg besitzen wir für den Osthallstattkreis einen gefäßtragenden Wagen, wie er sonst im nördlichen und mittleren Italien zu finden ist. Da wir so gut wie keine Bronzegefäße in den Gräbern der Kalenderbergkultur besitzen, dürfen wir hier m. E. dieselben in Keramik umgesetzt erwarten. Uns stehen für eine solche Umsetzung zwei keramische Fundensembles aus reich ausgestatteten Gräbern der Kalenderbergkultur aus Niederösterreich zur Verfügung (Langenlebarn und Gemeinlebarn). Vermutlich wurden daher diese Kultwagen - wie Strettweg (A) mit Figurenbesatz - in Ton nachgebildet bzw. auf eine organische Unterlage wie z. B. auf einen aus Holz hergestellten Wagen eingeklebt. In diesem Bildmaterial lässt sich wohl eine Schilderung von Kulthandlungen bzw. Prozessionen oder Mythen erkennen, die sich m. E. auch in den Darstellungen der Situlenkunst sowie auf den Zisten aus dem Kröllkogel von Kleinklein (A), aber auch in den figuralen Darstellungen der Kalenderbergkultur wiederfinden - nur jeweils in einem anderen Kunstmittel ausgedrückt.
\end{abstract}

\section{Schlagwörter}

Osthallstattkultur, Keramikfiguren, Kultwagen, Prozessionen 


\section{Einleitung}

Wir besitzen im bronzenen Kultwagen von Strettweg für den Osthallstattkreis einen Kultbzw. Ritualwagen (Woytowitsch 1978, 54) und zwar einen gefäßtragenden Wagen, wie er sonst vor allem im nördlichen und mittleren Italien zu finden ist, wobei hier neu ist, dass die in Durchbruchstechnik hergestellte zyprische Szenerie durch Kleinplastiken ersetzt wird (ebd., 56 ff.). An Motiven spielen dabei das Pflügen, der Zweikampf, die Jagd sowie der Hieros gamos, aber auch Tiere und Mischbzw. Fabelwesen eine Rolle, die sich allerdings auch in den getriebenen Bildern der Situlenkunst wiederfinden (Eibner 2012; 2015). Aber auch das Prinzip der zwei Ebenen dieser Kesselwagen, einer figurierten unteren und einer oberen zur Aufnahme des Gefäßes (Woytowitsch 1978, 57 f./Nr. 125, 58 ff./Nr. 127, 61 f./Nr. 131), ist hier durch den spiegelbildlich beidseits die Statue begleitenden Prozessionszug sowie durch die große, nackte weibliche Statuette in der Mitte des Wagens gegeben. Sie trägt ein mit den Händen gestütztes Gefäß am Kopf, das zusätzlich noch durch die sich überkreuzenden tordierten Verstrebungen gehalten wird (ebd., $56 \mathrm{f}$.). Nachdem es in der Kalenderbergkultur weitere Figürchen aus Bronze bzw. aus gebranntem Ton (= Keramik) gibt, die vom Motiv her ebenfalls hier anzuschließen sind, stellt sich die Frage, wo diese montiert gewesen waren, nachdem sich an deren Beinen Nietlöcher bzw. Teerklumpen zum Einsetzen in eine Unterlage gefunden haben.

\section{Zum Kultwagen von Strettweg}

Der Kultwagen mit seinen Bronzefiguren ist ein herausragendes Kunstwerk des Osthallstattkreises (Kruta 1993, 241 ff. mit Abb. 203-205) und stammt aus Strettweg in der Steiermark (Egg
1996), aus der Murtalregion - „Aichfeld/Murboden" (A) -, wo durch die Initiative des Arbeitskreises Falkenberg (Arbeitskreis Falkenberg (Hrsg.) o. J. [2015]) inzwischen wieder Grabungen stattfinden ${ }^{1}$. Diese haben im „Bleikolmhügel" in Waltersdorf (A) sogar ein phönizisches Glasgefäßfragment zutage gefördert und damit die weitreichenden Kontakte und Beziehungen dieser Region in der Hallstattzeit aufgezeigt (Tiefengraber, S. u. G. o. J. [2015], 51 ff. mit Abb. 49; vgl. zu den „internationalen“ Beziehungen auch Eibner 2016a, bes. 165 ff./Punkt 2. u. 176 ff./Punkt 3).

Die alles überragende, zentrale, nur mit einem Gürtel bekleidete nackte Frauenfigur mit erhobenen Armen stützt und hält mit beiden Händen ein verziertes Becken, das sie am Kopf trägt. Sie selbst steht auf einem elfspeichigen Rad in der Mitte eines Prozessionszuges, wobei sich der Zug spiegelbildlich symmetrisch um sie gruppiert, d. h. janusköpfig - nach beiden Seiten hin - agierend: Zunächst führen zwei, nicht näher geschlechtlich differenziert wiedergegebene Menschen - wohl Opferdiener - zwischen sich einen Hirsch am Geweih. Hinter diesen folgt ein ebenfalls nacktes Menschenpaar nach, wobei der Mann in der hoch erhobenen Hand ein Beil schwingt, während die Frau durch Schmuck ausgezeichnet ist. Nach der anderen Seite hin sind spiegelbildlich nochmals dieselben Paare angeordnet. Seitlich dieses Opferzuges (? - wegen des hocherhobenen Beiles in der Hand des nackten Mannes ${ }^{2}$ ) schirmen Reiter, ausgestattet mit Ovalschild und Lanze (Tiefengraber, S. u. G. o. J. [2015], 44-bei den Nachgrabungen gefunden), den Zug gegen die "Öffentlichkeit“ - vermutlich gegen die Prozessionsteilnehmer bzw. die Festteilnehmer - ab bzw. begleiten diesen (Kašuba 2011, 244 ff.-Prozessionsszenen) $)^{3}$. 


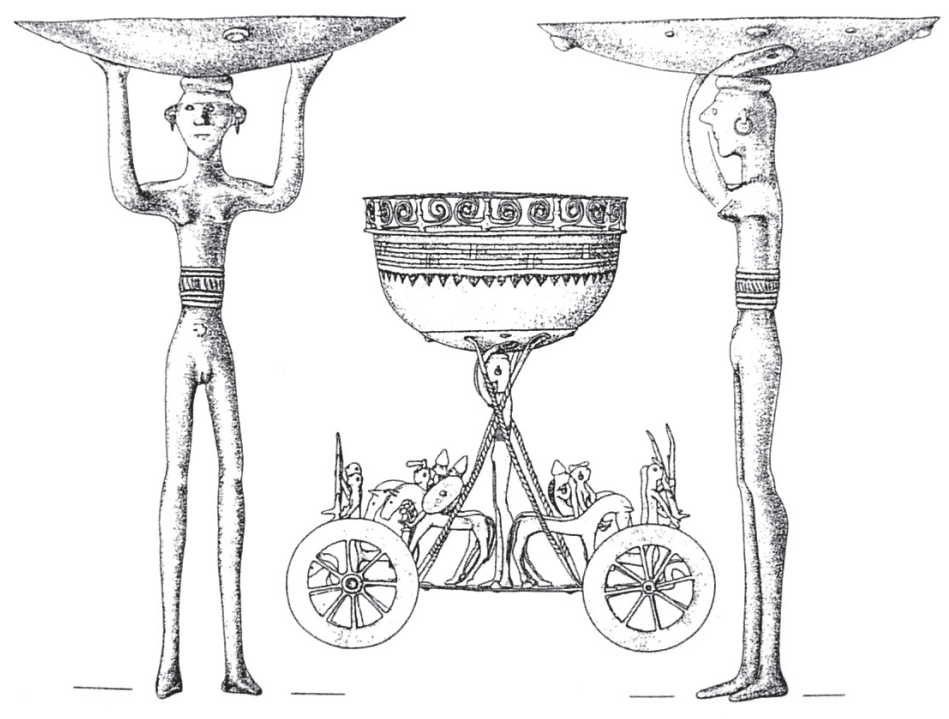

Abb. 1. Der bronzene Kultwagen mit zentraler nackter Frauenfigur aus dem Tumulus 1 von Strettweg, Steiermark (A) (nach Egg 1996).

\section{Vergleichsbeispiele aus dem Osthallstattkreis}

\subsection{In Bild und als Plastik}

Ähnliches ist auch Bild überliefert, auf der Ziste XIII aus dem Kröllkogel von Kleinklein in der Steiermark (A) (Abb. 2) (Egg 2013; Eibner 1993): Hier wird die Prozession beidseits ebenfalls von einem Reiterzug begleitet, wobei nur ein einziger behelmter Reiter als solcher vollständig dargestellt und erkennbar ist, während die anderen nur symbolhaft - Pferdekörper mit herunterhängendem Menschenbein - wiedergegeben sind. Allerdings lässt sich $\mathrm{m}$. E. Anfang und Ende des großen Frieses, über dem sich noch ein zweites schmäleres mit abwechselnden Gruppen von Vierfüßlern und Vögeln in S-Gestalt befindet, gut daran erkennen, dass an dieser Stelle, obwohl der zylindrische Zistenkörper eine zweimalige Nietung aufweist, zwei groß wiedergegebene Wasservögel, die jeweils den oberen, real aber seitlich die Prozession begleitenden Tierzug anführen und beenden, hier zusammenstoßen, wobei sich unter diesen Tieren ebenfalls zwei symbolische Reiterfiguren befinden (Eibner 1993). Diese großen Vögel sind sicher als Hinweis für Beginn und Ende des Zuges anzusehen, denn die sonst üblicherweise bei Abrollungen der Ziste als Anfang wiedergegebene zweite Schnittstelle zeigt hier eine symbolische Reiterfigur, die über diese Nietung hinwegreicht in den Beginn der figuralen Darstellung ${ }^{4}$ mit den Frauen, was m. E nicht zu einem Ende und Anfang einer szenischen Wiedergabe passt (Egg 2013, Beilage 23). Dieser große Fries selbst wird durch drei Aulosbläserpaare in Einzelszenen bzw. „Erzählungen" unterteilt: Die erste zeigt drei Büsten mit erhobenen Armen $^{5}$, dazwischen eingestreut sind Rinder, deren Gehörn kreisförmig gebildet ist, sowie weitere Vierbeiner - auffallend ist dabei die immer wiederkehrende Dreizahl (Eaciak - Markiewicz 2013, 538). Das zweite Bild 


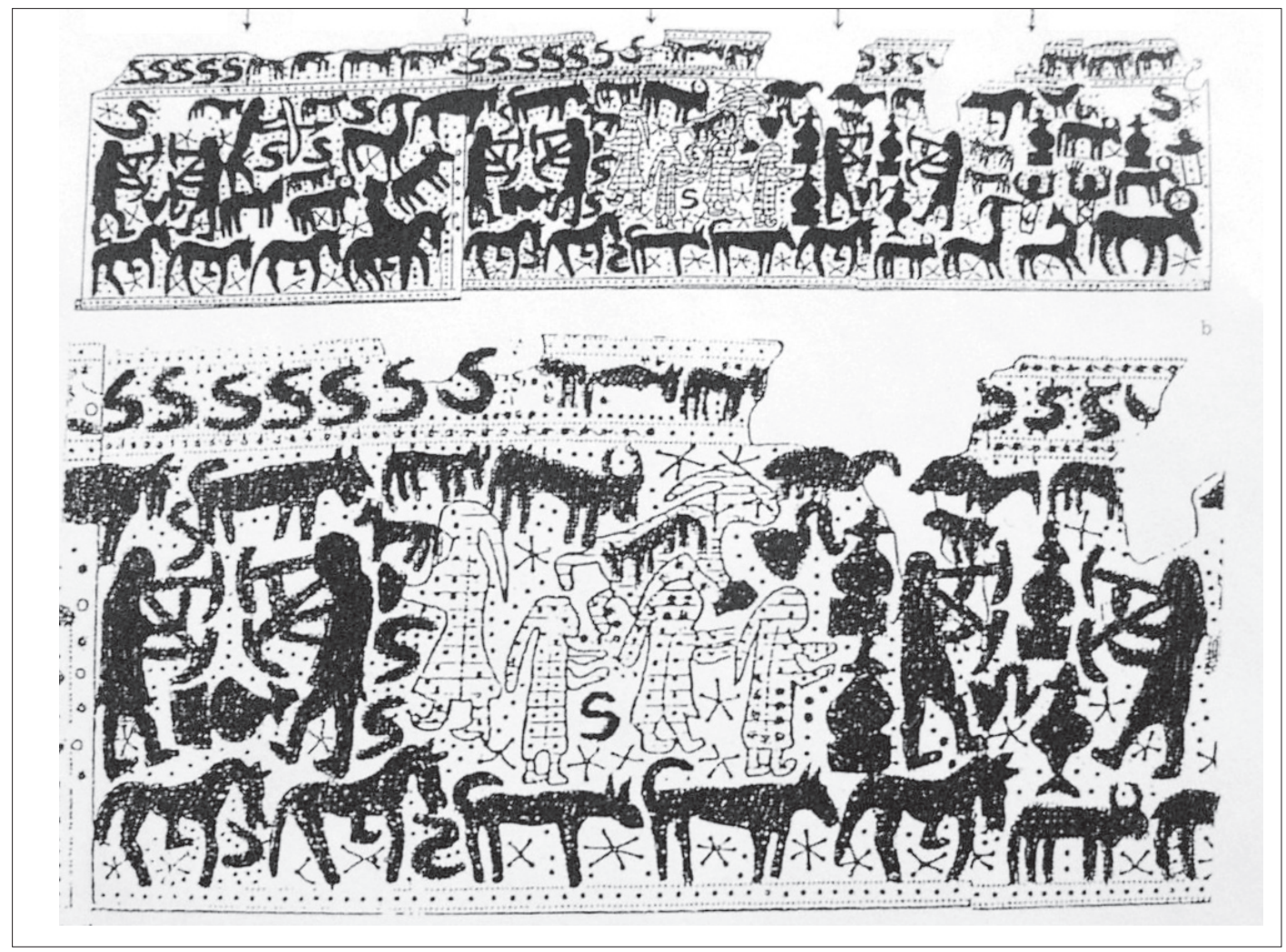

Abb. 2. Die Bronzeziste XIII aus dem Kröllkogel von Kleinklein, Steiermark (A) (nach Eibner 1993; vgl. auch Egg 2013, Beil. 23).

gibt eine „Prozession“ von drei nach rechts gehenden Frauen mit waagrecht vorgestreckten Armen wieder, wobei eine weitere Frau quasi mehr oder weniger schräg über den anderen wiedergegeben ist - sie hält ein Gefäß, einen Becher, in Händen und in ihrem Körper, im Gewand, eingeschrieben ist ein Tier (Abb. 2), als ob sie damit schwanger wäre ${ }^{6}-$ sie könnte als Anführerin gelten, die den Frauen/Mädchen voranschreitet. Die vorgestreckten Arme der drei Frauen scheinen dabei etwas zu tragen, möglicherweise einen Stoff, ein Gewand, das man der Gottheit weihen wollte - zum Teil erfolgte ja die Anleitung der Mädchen zum Spinnen und Weben im Tempel ${ }^{7}$, zu Fertigkeiten, ohne die eine Frau ihren Haushalt nicht führen und der Oikos nicht bestehen konnte
(Eibner 2000/2001, 108 ff./Punkt 1.1, bes. 114 f. u. 132). Eine fünfte Frau, ebenfalls mit einem Becher in Händen, dem linken Aulosbläserpaar zugewandt, scheint diesen - mit einem Trank gefüllt - den Musikanten anbieten zu wollen. Das dritte Bild stellt eine Jagdszene dar: Hier steht ein nackter, ithyphallischer Bogenschütze auf einem Huftier und richtet seinen Pfeil gegen den vor ihm befindlichen Steinbock (?). Nach dem Schwanz und den säulenförmigen Beinen zu urteilen ist es vermutlich ein Rind (?), auch weil es vier Füße zeigt, im Gegensatz zu den zweibeinigen Pferden der Reitersymbole, wobei die beiden davor gehenden Vierfüßler ebenfalls Rinder sind, damit wären es also insgesamt wieder drei gleichartige Tiere. Gegen das vordere Bläserpaar hin, den oberen Friesabschluss zu, 


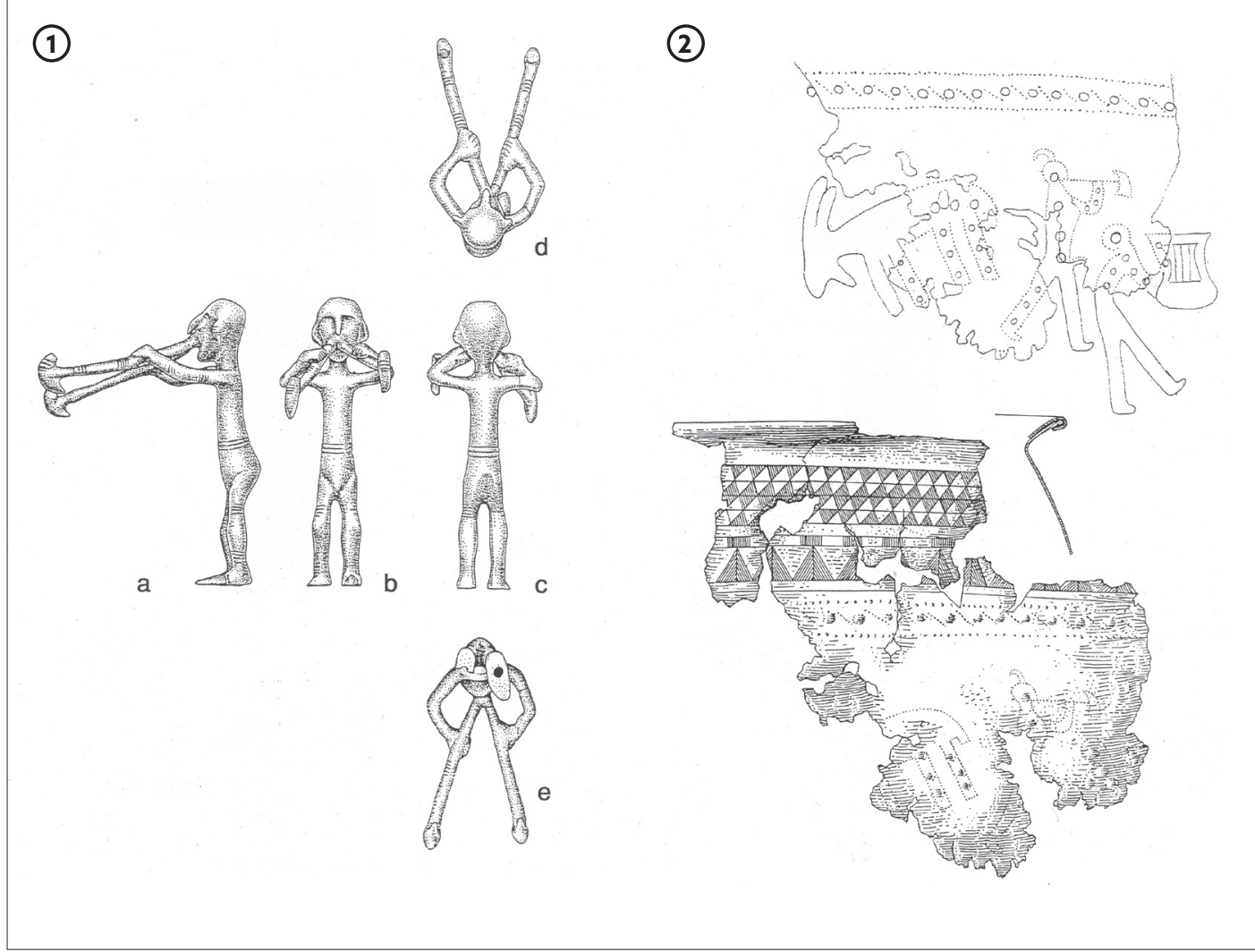

Abb. 3. Aulosbläser als Statuette und im Bild.

1 - Das Bronzefigürchen von Százhalombatta, Einzelfund, aus der Nekropole bei Budapest (H) (nach Eibner 1999, 39/Abb. 1: a-e)

2 - Ein bronzenes Gefäßfragment aus der Býčí skála Höhle bei Brno, Mähren (CZ), mit der Darstellung zweier Musiker in feiner Punkttechnik - ein Aulosbläser und ein Leierspieler (nach Eibner 1999, 47/Abb. 3).

sind dann nochmals drei Vierbeiner sowie drei Wasservögel erkennbar.

Ein weiteres Bronzefigürchen, das durchlochte Füße aufweist - in einem steckt noch ein Niet - und damit anzeigt, dass es irgendwo fix montiert gewesen sein musste (Abb. 3: 1), könnte möglicherweise auch auf einem solchen Wagen gestanden haben - als Musiker, denn das Figürchen aus Százhalombatta, aus der Nekropole am Donauknie bei Budapest $(\mathrm{H})$, stellt einen Auslosbläser dar und würde damit als Statuette an die Darstellungen der Aulosbläser von Klein- klein (A) (Abb. 2) und der Býčí skála-Höhle bei Brünn (CZ) (Abb. 3: 2) anzuschließen sein (Eibner 1999). Eventuell als hier zugehörig ist auch das Bruchstück - der Oberkörper - eines bronzenen Hantelkämpfers ${ }^{8}$ anzusehen.

\subsection{Plastiken aus Keramik in der Kalen- derbergkultur}

Aus diesem Bereich kennen wir jetzt Figuren aus Keramik, die vom Motiv her den Bronzestatuetten des Strettweger Wagens gleichen 
(1)

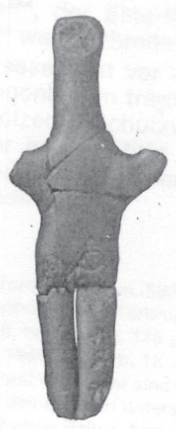

$28 / 3$

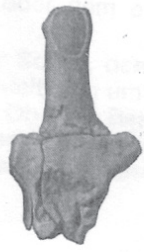

$28 / 9$

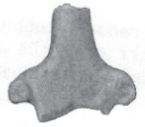

$28 / 15 \mathrm{~A}$

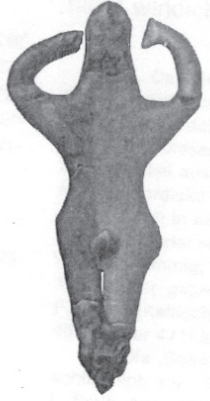

$28 / 10$

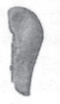

$28 / 16 \mathrm{~A}$

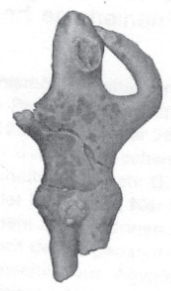

28/11A

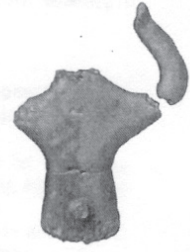

$28 / 12$

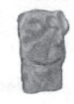

$28 / 17 \mathrm{C}$

(2)

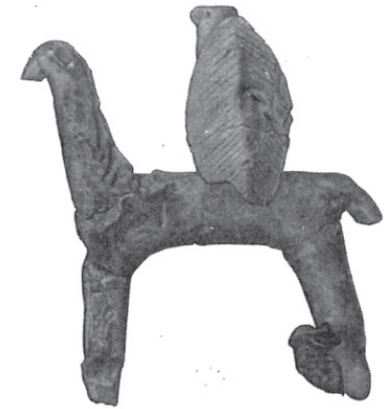

a

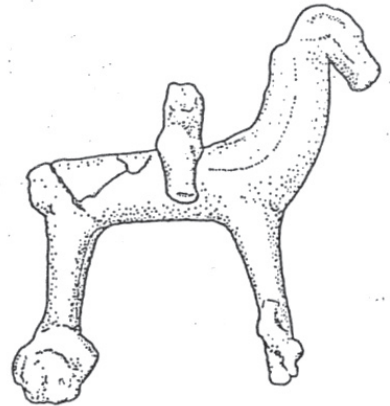

b

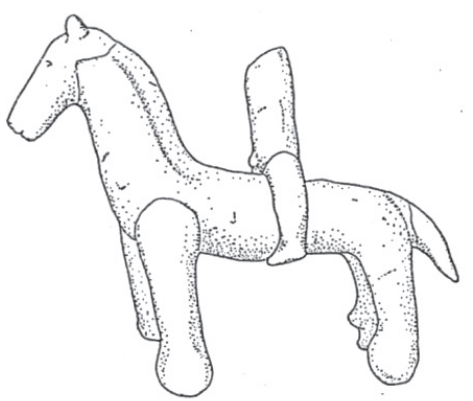

C

Abb. 4. Anthropomorphe und zoomorphe Keramikfigürchen aus zwei reich ausgestatteten Gräbern der Kalenderbergkultur aus Niederösterreich (A).

1 - Ithyphallische bemalte Männerfiguren mit verbreitertem Becken und mit zum Kopf erhobenen Armen sowie geschlechtslos wiedergegebene Figuren aus dem Tumulus 3 von, Langenlebarn (nach Preinfalk 2003, 86/ Abb. 39).

2 - Reiterfiguren aus der Kalenderbergkultur (nach Preinfalk 2003, 89/Abb. 41).

a - Mit Ovalschild aus dem Tumulus 3 von Langenlebarn; b - Aus dem Tumulus I von Gemeinlebarn;

c - Aus dem Kleinen Hügel von Großmugl.

3 - Mit rockartigem Gewand, aus Keramik: Mit am Kopf festgeklebten Gefäßen sowie eine Frau mit durch Ringe gelängtem Hals aus dem Tumulus I von Gemeinlebarn (A) (nach Kromer 1958).

4 - Frauenfiguren mit rockartigem Gewand, teilweise mit Gefäßen auf dem Kopf, Pferde sowie je ein Reiter und ein Hirsch aus dem Tumulus I von Gemeinlebarn (nach Nebelsick 1997, 60/Abb. 19 bzw. nach Kromer 1958). 
(3)

(4)
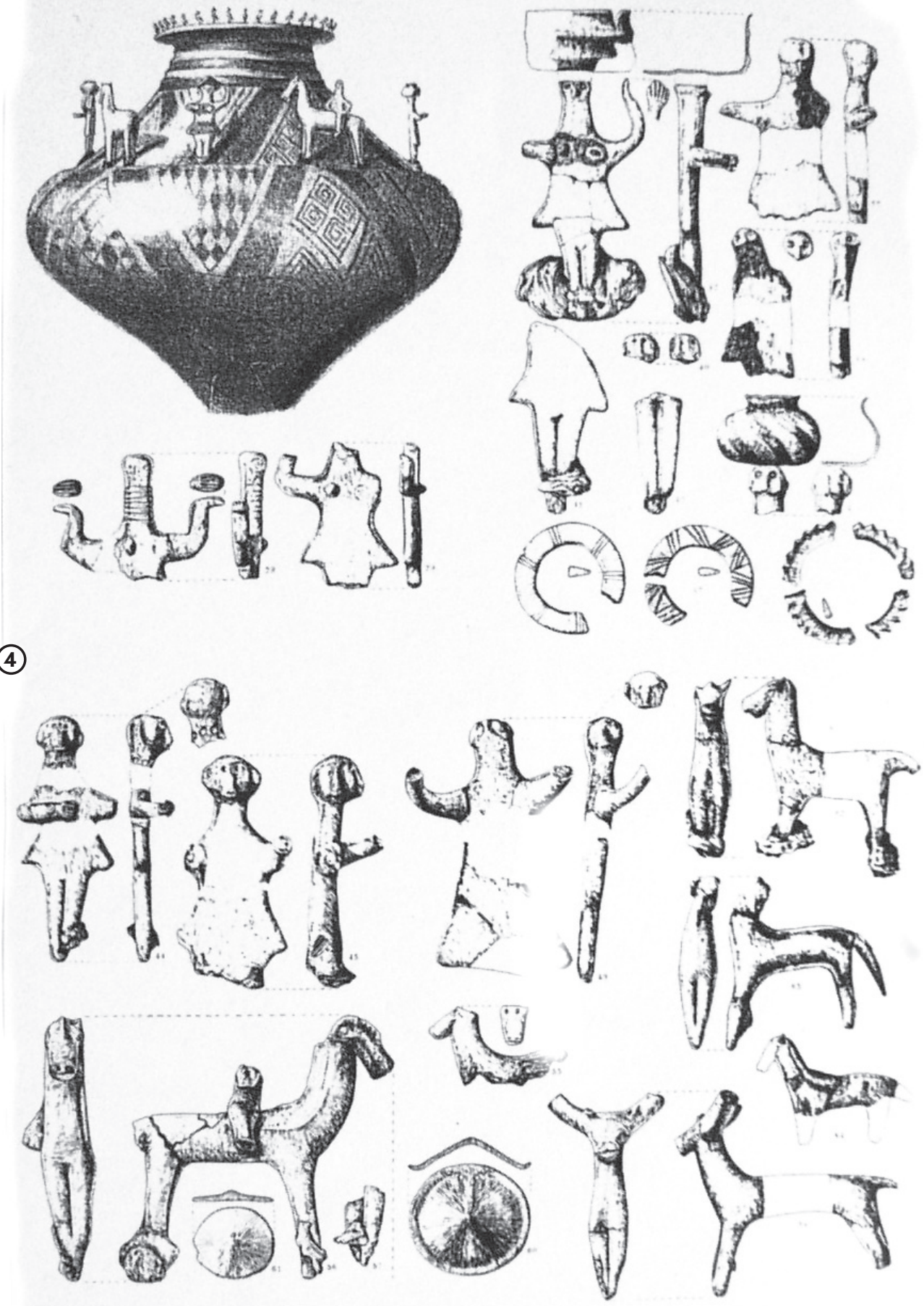
(1)

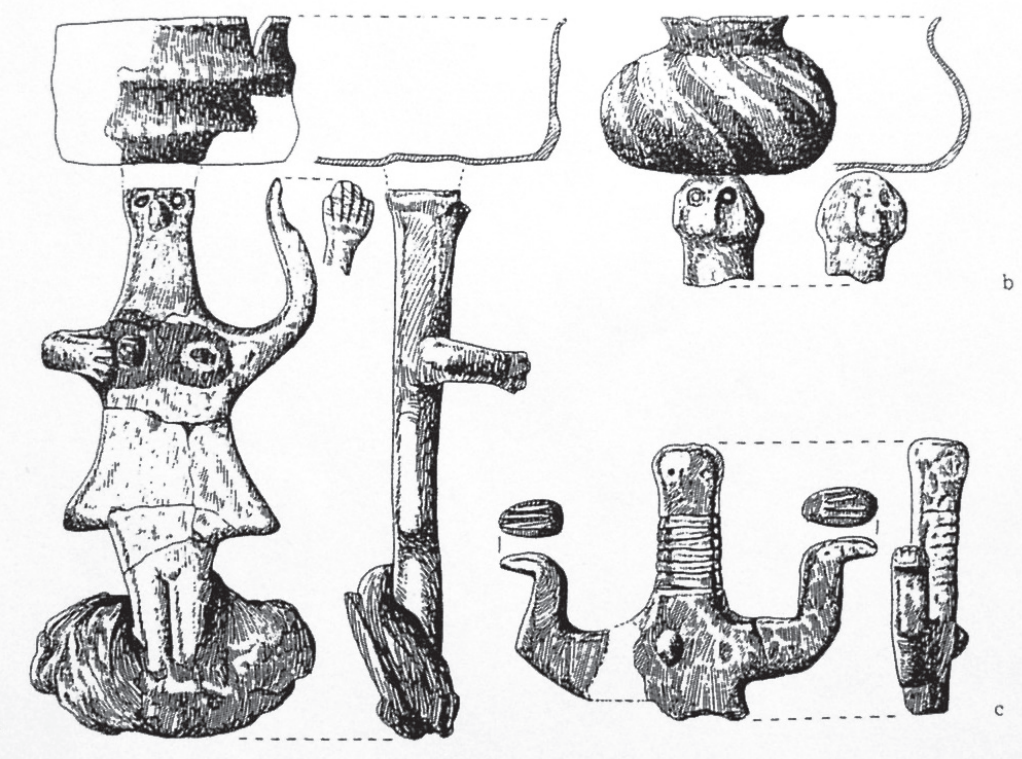

(2)
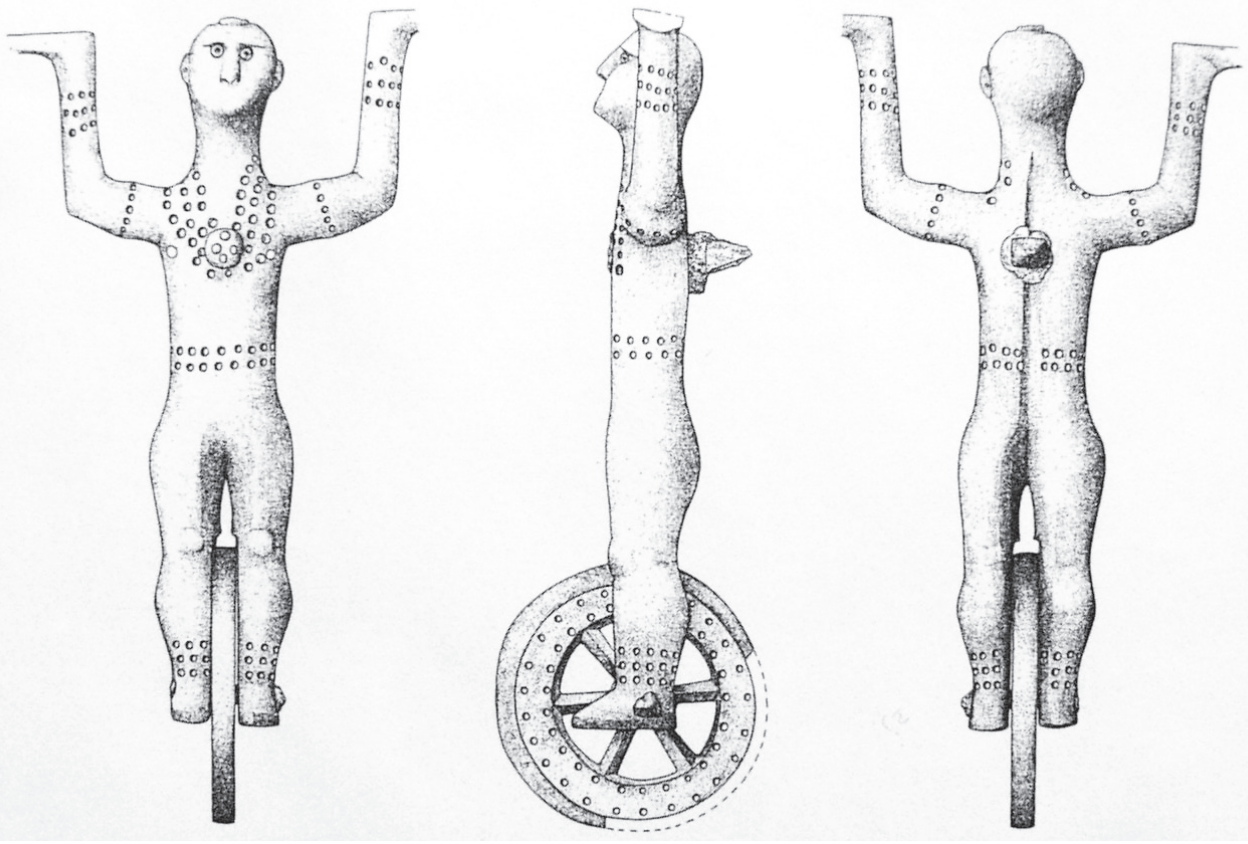
(3)

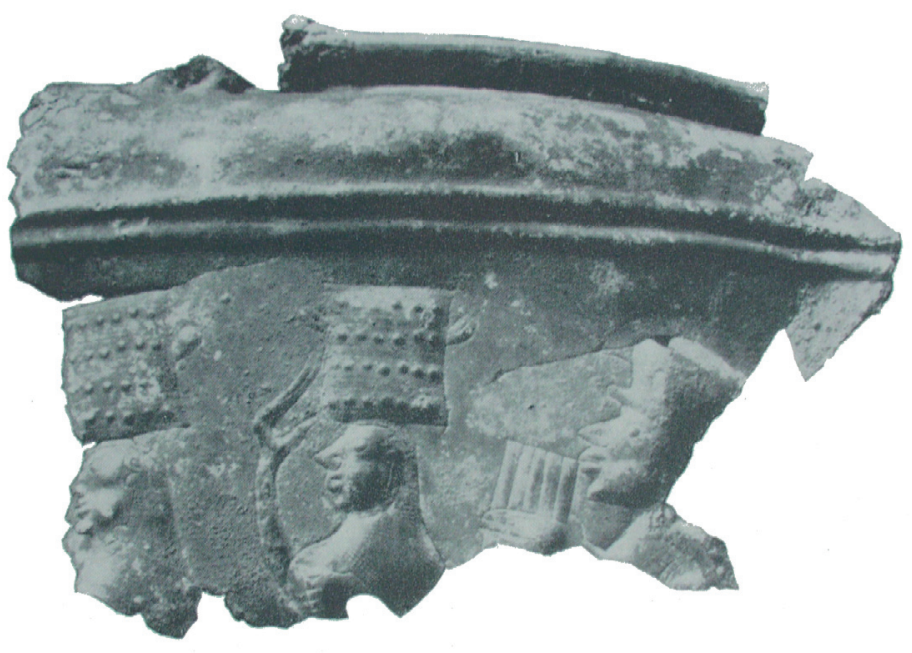

(4)
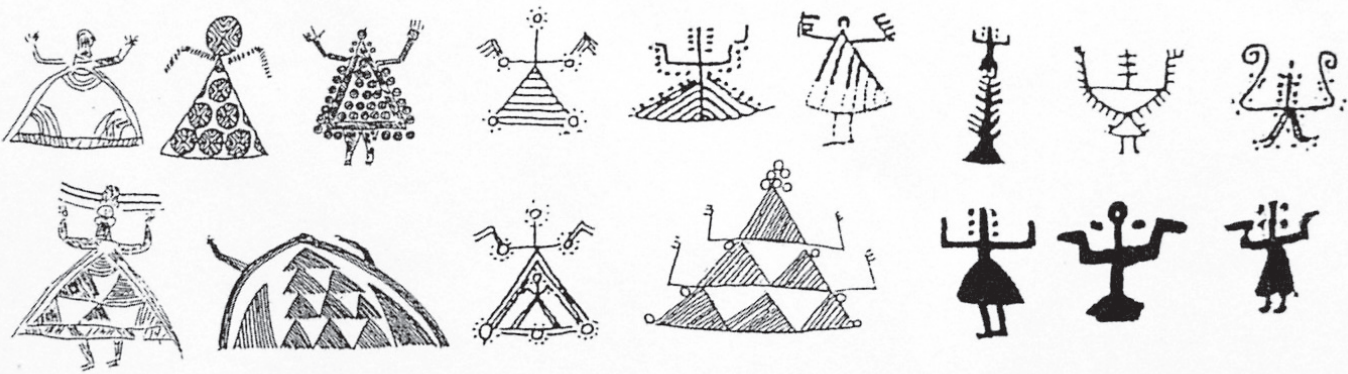

Abb. 5. Frauenfiguren mit erhobenen Armen, Gefäße am Kopf balancierend.

1 - Mit rockartigem Gewand, aus Keramik: Mit am Kopf festgeklebten Gefäßen sowie eine Frau mit durch Ringe gelängtem Hals aus dem Tumulus I von Gemeinlebarn (A) (nach Kromer 1958).

2 - Nackt, aus Bronze: Mit Gürtel und Schmuck, darin Koralleneinlagen - stützen sie, mit und ohne Gefäß am Kopf, auf Rädern stehend die "Kline" aus dem Fürstengrab von Hochdorf-Eberdingen, Baden-Württemberg (D), ab (nach Biel 1985).

3 - Situlenfragment, aus Bronzeblech getrieben, aus dem Grab 23 von Welzelach, Matrei in Osttirol (A): Mit Frauen, die Zisten am Kopf balancieren, und nachfolgendem Syrinxbläser (nach Lucke - Frey 1962, Taf. 60: Kat. Nr. 44).

4 - Frauendarstellungen mit dreieckigem Gewand auf Keramikgefäßen des Osthallstattkreises, eingeritzt oder in Graphit gemalt [unten links die dritte Figur = Maiersch, Grab 89 (A) (nach Berg 1962)] (nach Eibner 2000/2001, 123/Abb. 11). 
bzw. den figuralen Darstellungen in Punkt-Buckel-Technik auf der Ziste XIII von Kleinklein (beide: A) (Abb. 1 u. 2): Dazu gehören Frauen mit dreieckigem Rock und erhobenen Armen, die auch Gefäße am Kopf balancieren (Abb. 5: 1), ithyphallische Männerfiguren sowie geschlechtslos wiedergegebene Statuetten (Abb. 4: 1) (Preinfalk 2003, 85 ff./Punkt 7.3.6.3.4), aber es finden sich auch Hirsch- und Pferdefiguren (ebd., 80 ff./Punkt 7.3.6.3.3) sowie Reiter, einmal auch mit Schild ${ }^{9}$ (Abb. 4: 2) (ebd., 88 ff./Punkt 7.3.6.3.5). Diese Figuren weisen an den unten zapfenförmig zusammengeführten Beinen Birkenteerklumpen auf, was bedeutet, dass sie mittels dieses Klebstoffes irgendwo eingesetzt und verklebt gewesen waren (Abb. 4: 1-2; 5: 1) (Sauter - Graf - Hametner - Fröhlich - Neugebauer - Preinfalk 2002; Preinfalk 2003, bes. 85 mit Anm. 524 u. 525). Sie haben sich in dem reich ausgestatteten Wagengrab von Gemeinlebarn, Tumulus I (A) Neugebauer 1997, 165 ff., bes. 166-bei der Nachuntersuchung fand sich eine Pferdebestattung und ein Brandrest mit Achsenhülsen eines Wagens), sowie in Langenlebarn, Tumulus 3 (A) (ebd., 168 ff., bes. 171-hier wird von einem „Großgefäß mit plastischer Verzierung“ gesprochen; vgl. dazu Preinfalk 2003, 79 ff./Punkt 7.3.6.3), gefunden: Im ersteren waren es sechs Pferde und eine Hirschfigur (Abb. 4: 3) (ebd., 83/Abb. 37: unten; 89/Abb. 41: 2; 91/Abb. 42: unten-Reiter u. Hirsch), im letzteren sechs Pferdefiguren und das Pferd mit Reiter (Abb. 4: 2) (ebd., 83/Abb. 37: oben; 89/Abb. 41: 1-Reiter mit Schild) sowie mindestens sieben anthropomorphe Plastiken, weil sich sieben Köpfe gefunden hatten, wobei die größte der Figuren (Fn. 28/3) sowie noch zwei weitere (Fn. 28/9 ist etwa gleich groß wie 28/3) geschlechtslos wiedergegeben sind (Abb. 4: 1). Für sie charakteristisch sind dabei ein überlanger Hals, seitlich angedeutete Ohren, nach oben gestreckte (Fn. 28/3 u. 9) oder nach vorne gebogene Arme (Fn. 28/15A) und ein abgeplatztes Gesicht. Drei weitere Figuren sind allerdings ithyphallisch wiedergegeben (Fn. 28/10-nur wenig kleiner als 28/3; 28/11A u. 12): Auffällig bei ihnen ist die verschiedenfärbige Bemalung der Körper, wobei sich an der rechten Körperhälfte eine schwarze findet, die linke hingegen eine Rotfärbung aufweist ${ }^{10}$, alle haben jedoch die Arme nach oben zum Kopf erhoben, während zwei der Figuren ein verbreitertes Becken zeigen (Abb. 4: 1).

Die besten Parallelen zu den anthropomorphen Figürchen finden sich wieder in Gemeinlebarn (A), wo mindestens vierzehn davon im Tumulus I gefunden wurden, die im Allgemeinen etwas größer und von gröberer Machart sind als jene in Langenlebarn, Tumulus 3 (Preinfalk 2003, 85 f. mit 87/Abb. 40; 91/Abb. 42). Auch hier lassen sich geschlechtslose und eindeutig geschlechtlich zuordenbare Statuetten erkennen, allerdings nur weibliche mit deutlich ausgebildeten Brüsten. Hier ist besonders auffällig, dass jeweils die linke Brust - nach dem Brand - abgebrochen ist, wahrscheinlich intentionell, weil bei keiner der Frauen beide Brüste erhalten sind ${ }^{11}$. Auch hier findet sich bei einigen der Plastiken eine Bemalung und zwar eine schwarze bei der linken Körperhälfte bzw. eine rote bei der rechten (Abb. 4: 3). Sie zeigen aber $\mathrm{m}$. E. eine rockartige Bekleidung, wie eine solche alle Frauenfiguren des Osthallstattkreises tragen - als dreieckiger Umriss wiedergegeben, gleichgültig ob aus Ton gebildet (Abb. 5: 1) oder dargestellt in Graphitmalerei und Ritztechnik (Abb. 5: 4) - und keine Verbreiterung des Beckens (vgl. Eibner 2000/2001, 108 im Gegensatz zu Preinfalk 2003, 85) ${ }^{12}$. Aber hier variieren die Armhaltungen stärker: Nach vorne gestreckt oder nach oben erhoben (Eibner 2000/2001, 122 ff./Punkt 2.), wobei diese Geste auch zur Abstützung von am Kopf getragenen Gefäßen dient ${ }^{13}$ (vgl. Abb. 5: 1-3) (ebd., 118 ff./ Punkt 1.3.1), doch auch nach außen abgewinkelte Arme mit nach oben gedrehten Handflä- 
chen kommen vor (Preinfalk 2003, 87/Abb. 40: 28; 91/Abb. 42) ${ }^{14}$. Die zusammenlaufenden Beine ergeben dabei ebenfalls einen Einsteckzapfen und sind von einem Teerklumpen umhüllt, sodass man an eine Montage auf organischem Material zu denken haben wird, nachdem sich außer des einen fraglichen Gefäßes bei erneuerter Durchsicht von F. Preinfalk (2003, 85 mit Anm. 523-526) kein weiteres mit Lochungen am Hals-Schulter-Umbruch gefunden hat.

\section{Diskussion der ursprünglichen Anordnung der Figürchen}

Bisher war die Rekonstruktion dieser Figuren derart gewesen, dass man sie auf der Schulter eines Kegelhalsgefäßes eingesetzt hatte (Abb. 4: 3), doch neuere Untersuchungen der Gemeinlebarner Figürchen zeigten, dass diese Rekonstruktion möglicherweise nicht stimmen könnte, weil sich auf der Gefäßschulter - im heutigen Zustand - zum Einsetzen derselben keine Löcher mehr feststellen ließen ${ }^{15}$. Außerdem wurden jetzt bei den neueren Grabungen in Langenlebarn (A), in Niederösterreich gelegen wie Gemeinlebarn, ebenfalls solche Figürchen, vor allem Reiterfigürchen, gefunden, deren Lage einen Zusammenhang ergaben, als wären sie auf einer organischen Unterlage montiert gewesen (Preinfalk 2003, 85; vgl. dazu Neugebauer 1997, 169/171-mit mindestens 15 Großgefäßen in noch unrestauriertem Zustand), eventuell auf einem hölzernen Wagen (vgl. dazu auch Preinfalk 2003, 92 mit Anm. 599) ${ }^{16}$.

Analysiert man jetzt alle diese Figürchen aus beiden Grabhügeln, so sind Frauenstatuen überliefert - mit erhobenen Armen, die aber auch Gefäße am Kopf balancierten -, ebenso auch nackte Männer - mit nach oben erhobenen bzw. nach vorne ausgestreckten Armen -, wobei diese anthropomorphen Statuettentypen eine Bemalung aufweisen - für die eine Körperhälfte eine schwarze, für die andere eine rote -, aber auch geschlechtslos wiedergegebene Figuren kommen vor. Dazu gesellen sich noch Pferde, ebenso sind Reiter nachgewiesen - einer ist dabei mit einem ovalen Schild ausgestattet - und einmal ist darunter, nach dem erhaltenen Geweih zu schließen, auch eine Hirschfigur. Viele dieser Figuren weisen zudem auf ihren miteinander verbundenen Füßen einen Teerklumpen auf, mit dem sie auf einer Unterlage aufgeklebt gewesen sein mussten ${ }^{17}$.

Vergleichen wir diese Keramikfigürchen jetzt mit den Bronzeplastiken auf dem Strettweger Wagen: So liegen hier ebenfalls geschlechtslos wiedergegebene Menschen vor - sie führen zwischen sich einen Hirsch am Geweih mit -, während hinter ihnen ein nacktes Menschenpaar nachfolgt, wobei der Mann in seiner erhobenen Hand ein Beil schwingt, soll er damit den Hirsch als Opfertier töten ${ }^{18}$ ? Seitlich wird dieser spiegelbildlich von der großen, in der Mitte stehenden, nackten Frauenstatue ausgehende Zug von mit Helm und Schild ausgerüsteten Reitern begleitet.

So kennen wir auch in der Situlenkunst aus Bronzeblech getrieben - Prozessionszüge (Eibner 2012; 2015) mit Frauen, die Gefäße am Kopf balancieren (vgl. Abb. 5: 3; Abb. 7) (Eibner 2015, 61/Taf. 3: b-c), und mit mitgeführten Opfertieren wie Rind und Widder (Abb. 7) (ebd., 61/Taf. 3: b) bzw. Widder und Hirsch (ebd., 61/Taf. 3: a). Auf der Ziste Sanzeno (I) wird allerdings eines dieser Tiere, dessen Kopf leider fehlt, von einem nackten Mann mit Beil und Lanze getötet (ebd., 65/Taf. 4: j) bzw. wird auf der Situla Certosa (I) auf der Stange ein getöteter Hirsch zum Fest herangetragen (ebd., 61/Taf. 3: b) und auf der Situla DürrnbergKranzbichl (A) eine Hirschkuh (ebd., 65/Taf. 4: 1). Der beidseits von Reitern begleitete Prozessionszug auf der Ziste XIII aus dem Kröllkogel von Kleinklein (A) ist allerdings in der älteren 

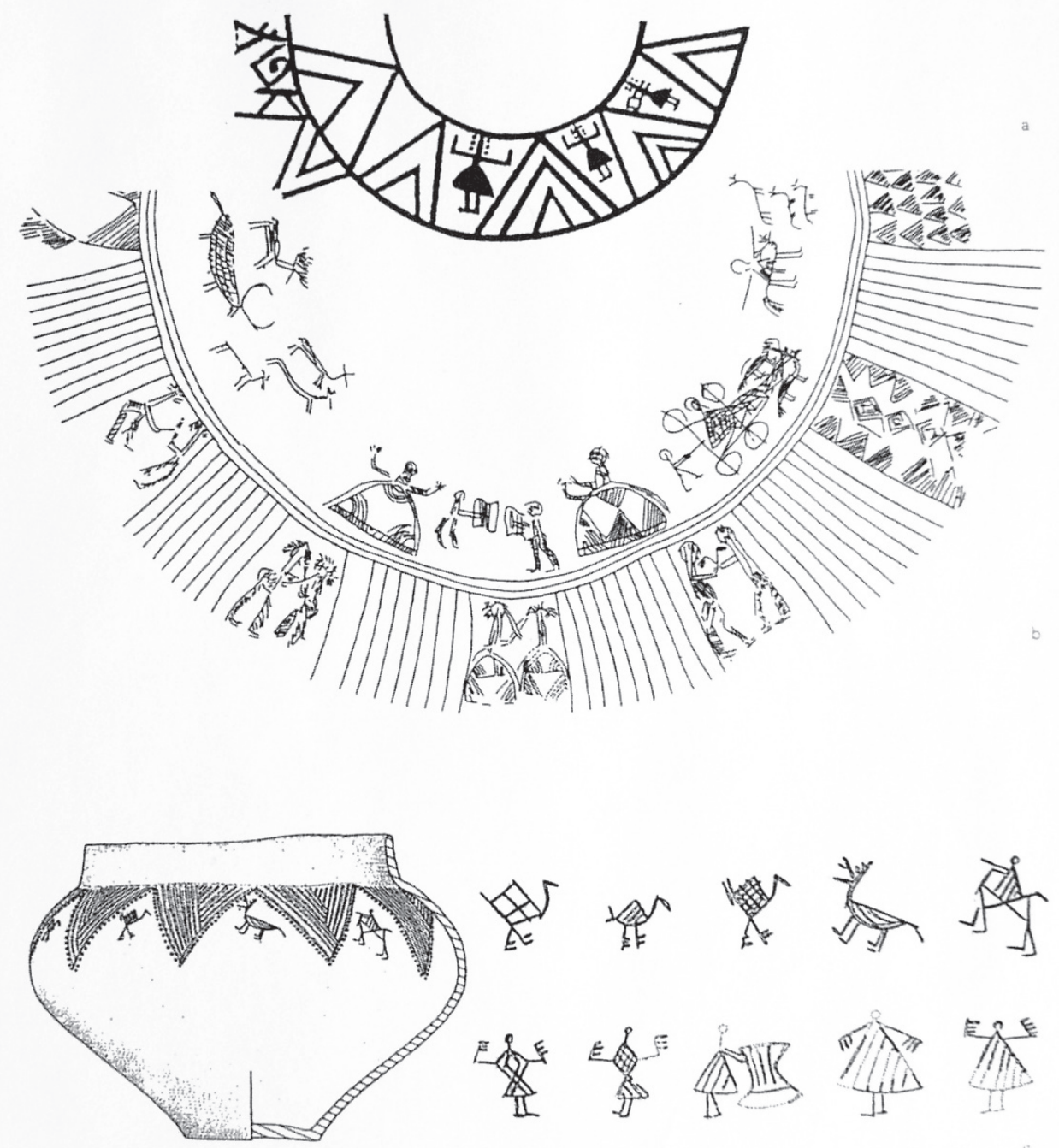

Abb. 6. Frauen mit erhobenen Armen, Reiter, Musikanten/Leierspieler und Tanzszenen - Wagenfahrt, Jagd auf Hirsch und Rind mit Hunden, Vögel - in Ritztechnik oder Graphitmalerei auf Keramikgefäßen der Kalenderbergkultur. Ist eine eigene Tafel?

a-aus Tumulus II von Janíky (SK) (nach Studeníková 1995);

b-aus Tumulus 28 von Sopron/Burgstall $(H)$ (nach Eibner-Persy 1980);

C-Grabgefäß aus Reichersdorf (A) (nach Neugebauer 1988). 


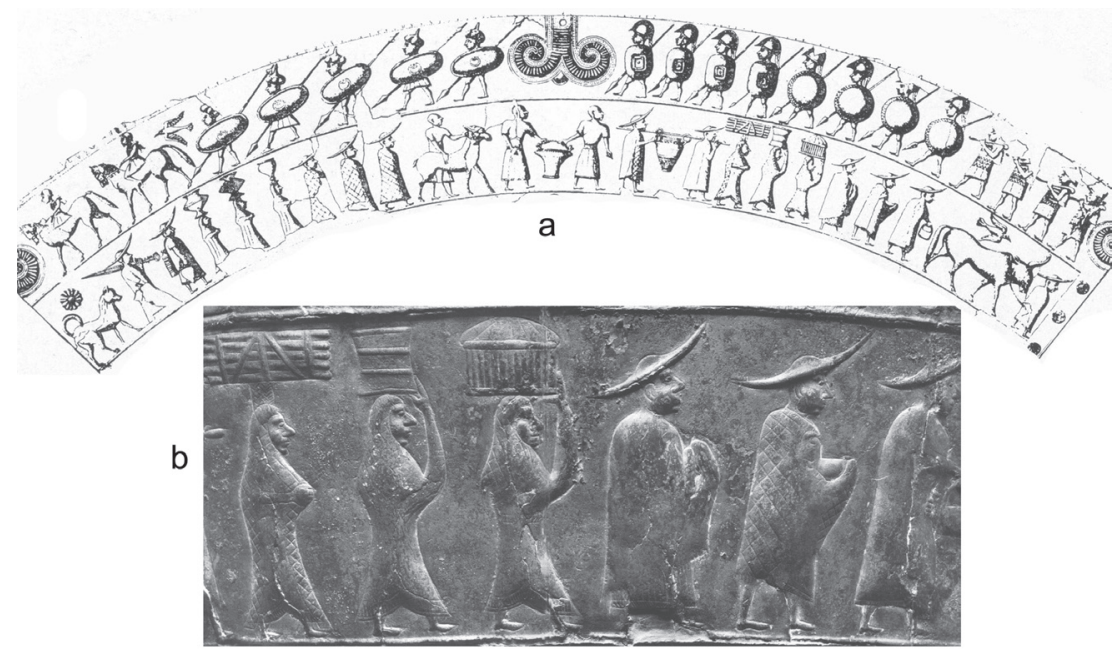

C

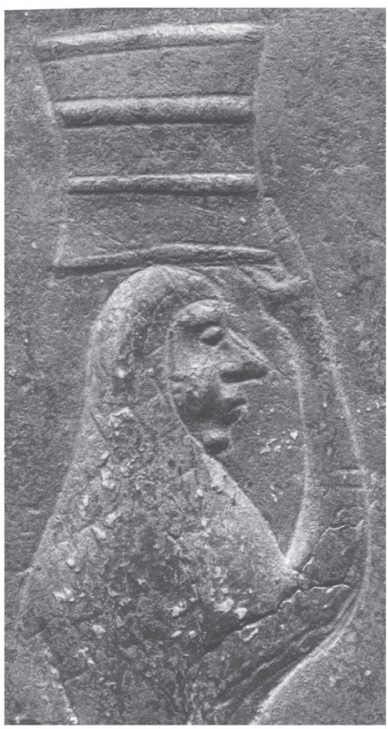

Abb. 7. Prozessionszüge mit Rind und Widder als Opfertiere auf der Situla Certosa aus Bologna, Grab 68 (I). a - Abrollung erstes und zweites Fries - nach Lucke - Frey 1962, Taf. 64: Kat. Nr. 4;

b u. c - Photo - nach Kastelic 1964, Taf. 24/25 (b - Ausschnitt aus dem ersten Prozessionszug, c - Detail daraus mit zweiter Frau mit Arm- und Fingerring). 
Punkt-Buckel-Technik ausgeführt: Hier gehen Frauen mit vorgestreckten Armen im Zug mit und auch ein ithyphallischer, auf einem Tier stehender Jäger mit Pfeil und Bogen findet sich dargestellt sowie Büsten, Ahnenfiguren (?) ( $E g g$ 2013, 466 f. mit Abb. 208) und Musiker - in dem Fall Aulosbläser als Bildtrenner zwischen den einzelnen Szenen (Abb. 2).

\section{Zusammenfassung}

Können wir aus diesen Vergleichen auf einen, dem Strettweger Kultwagen ähnlichen Wagen aus organischem Material, eventuell auf einen hölzernen Wagen mit figürlicher Ausstattung schließen?

Da wir so gut wie keine Mitgabe von Bronzegefäßen in den Gräbern der Kalenderbergkultur kennen, sodass wir hier $\mathrm{m}$. E. dieselben in Keramik umgesetzt erwarten durften, wie sich ja auch Tonsitulen - genauso wie Bronzesitulen - mit Teeranstrich finden lassen (Persy [Eibner] 1967; Chytráček - Chvojka - Egg - John - Kyselý - Michálek - Ritter - Stránská 2015) und so ist in Stillfried an der March (A) sogar eine Zungenphiale aus Keramik mit zur Gänze graphitierter Oberfläche überliefert, die man üblicherweise in Bronze gefertigt hat (Eibner 1974, Taf. 18: 22; vgl. dazu auch C. Eibner 2016, 18 f. mit Abb. 2).

So lässt sich als ein weiteres Beispiel für die Umsetzung eines Bronzeobjektes - eines gefäßtragenden Bronzewagens in Keramik - ein vierrädriger Tonwagen mit einem Kalenderberggefäß als Aufsatz aus Fertőendréd, Kom. Sopron (H) anführen (Kašuba 2011, 246/Abb. 7: 7; vgl. Gómóri 2002), dem ein gleichartiger tönerner Wagen mit einem aufgesetzten bemalten Gefäß aus Domasław, Grab 4270, Kr. Wrocław (PL) (Gediga 2010, 217 mit 203-205/Abb. 13-15; 208/Abb. 19; 2011), in der Randzone, der nordöstlichen Provinz der Hallstattkultur gelegen, an die Seite zu stellen ist (Gediga 2011, bes. 109 f. mit Abb. 17; Łaciak - Markiewicz 2013, 530 f. mit 535/Fig. 8; 528/Fig. 1: 3).

Uns stehen jetzt für eine solche Umsetzung zwei keramische Fundensembles aus reich ausgestatteten Gräbern der Kalenderbergkultur zur Verfügung: Das eine mit männlichen Figuren ausgestattet, stammt aus Langenlebarn (A), das andere mit weiblichen Statuetten aus Gemeinlebarn (A), wobei sich die weiteren Plastiken gleicherweise aus Pferden, Reitern und auch einem Hirsch zusammensetzen. M. E. liegen daher mehr oder weniger die gleichen Menschentypen und Tiere vor, die auch auf dem Kultwagen dargestellt sind - nur in Keramik ausgeführt.

\section{Ergebnis}

Vermutlich wurden daher diese Kultwagen wie Strettweg (A) mit Figurenbesatz - wie in Langenlebarn bzw. in Gemeinlebarn (beide: A) - oder mit Gefäßaufsatz - wie in Sopron (H) bzw. in Domasław (PL) - ebenfalls in Ton nachgebildet bzw. auf eine organische Unterlage wie z. B. einen aus Holz hergestellten Wagen eingeklebt, in Nachahmung der bronzenen Vorbilder.

Erkennen können wir in diesem Bildmaterial wohl eine Schilderung von Kulthandlungen bzw. Prozessionen oder Mythen (Abb. 1; 3: 1) (C. Eibner 2012), die sich m. E. auch in den Darstellungen der Situlenkunst (Abb. 7; 5: 3) (Eibner 2014; 2015) sowie auf den Zisten aus dem Kröllkogel von Kleinklein (A) (Abb. 2) (Eibner 1993; Egg 2013, bes. 470 ff.), aber auch in den figuralen Darstellungen der Kalenderbergkultur (Abb. 3: 2; 4: 1-3; 5: 1 u. 4; 6) (Eibner 1997; Nebelsick 1997, 125/Abb. 46-Kombinationsgruppen der figuralen Kunst; Eibner 2000/2001, 108 mit 109/Abb. 1; Gleirscher 2009) wiederfinden lassen - nur jeweils in einem anderen Kunstmittel ausgedrückt. 
1) Aufgrund geophysikalischer Prospektion konnte man jetzt das Kultwagengrab wieder lokalisieren als Tumulus I von Strettweg (Totschnig o. J. [2015], bes. 18 f. sowie Tiefengraber, S. u. G. o. J. [2015], 39 f.).

2) Vgl. dazu den nackten Mann, der auf der Situla Sanzeno dem Huftier ein Beil in den Rücken schlägt und mit der Lanze nachstößt: Eibner 2004, 628 f./Punkt 2.2.3.2 u. 630 f./Punkt 3.2 sowie 631 f./Punkt 4. mit Taf. 5: 1-2; Taf. 5: 4-9 u. 635/Tab.1: Jagdwaffen/Beil; 2015, 63 f./Kap. II.b mit Taf. 4: j, k; auch Dies. 2016a, bes. 168-170 mit Abb. 12-13; vgl. auch Tiefengraber, S. u. G. o. J. [2015], 43 f.

3) Alle diese Szenen zeigen uns die große Bedeutung der dargestellten Handlungen, die mit gemeinsamen Mahlzeiten zusammenhängen, wozu auch das Trinken bestimmter Getränke gehörte - letzteres wird durch die besondere Stellung von Kesseln und anderer großer Gefäße für Flüssigkeiten unterstrichen (vgl. Gleirscher Nothdurfter - Schubert 2002, 264 f.; Eibner 2016b, 74 mit Taf. 8: a, g-große Eimer u. 76 mit Taf. 9: d, e; Taf. 6: d; auch Taf. 9: f, g-Becken sowie 74 mit Taf. 8: a, f= Taf. 6: b-Beil; und hier Abb. 1 u. Anm. 2).

4) Allerdings zeigt sich hier im großen Fries die einzige komplett wiedergegebene Reiterfigur des Zuges, der die drei Bilderzählungen flankiert - als Anführer desselben. Hinter dem Reiter folgen dann nur mehr Pferde mit herunterhängendem Bein, also Reiter-Symbole nach, genau unter dem Jagdbild: Es sind deren drei bis zur Nietung und der Bildszene mit den Büsten - Ahnenfiguren (?) -, allerdings finden sich auch noch drei dieser Symbole davor, jeweils unter den beiden restlichen Aulosbläserpaaren. - Vgl. dazu die Büsten - auch bekleidet und mit Armen ausgestattet -, die sich in etruskischen und auch estensischen Gräbern finden (Aigner-Foresti 2010, 139 f. mit Anm. 58-65; vgl. dazu Egg 2013, 166 ff. mit Abb. 65 sowie 173 f.).

5) Vgl. dazu die Ahnenbüsten, die man bei etruskischen und römischen Begräbnissen mitgetragen hat - hier zunächst als „Wickelkinder“ angesprochen: Eibner 1993; vgl. dagegen Egg 2013, 466 f. mit Anm. 1589.

6) Eine ähnliche Darstellung kennen wir aus Maiersch, Niederösterreich (A) (Berg 1962, 36 mit Taf. 29: 1-Grab 89): Hier finden sich auf dem Hals eines Kegelhalsgefäßes eingeritzt, abwechselnd Frauen mit erhobenen Armen und in sich gestrichelte Dreiecke mit einer kleinen Delle auf der Spitze, wobei im Körper einer der Frauen - wiedergegeben im dreieckigen Rock - eine kleinere Menschenfigur ebenfalls mit erhobenen Armen eingeschrieben ist (Abb. 5: 4). Wäre diese Figur als Kind im Bauch der Frau zu werten, so ist damit die Frau als schwanger anzusprechen? Auf einer relativ neu gefundenen Situla aus Montebelluna (I) stehen sich zwei Spinnerinnen gegenüber, wovon die linke größere Frau schwanger zu sein scheint, da der Gürtel nicht in der Taille sitzt, sondern darunter (Eibner 2014; 2015, 71 ff./Kap. III.b mit Abb. 7: c; 2016b, 74 mit Anm. 5 u. Taf. 6: b).

7) Eibner 2005, 32 mit Anm. 13-15; Belanová - Čambal - Stegmann-Rajtár 2007. - Vgl. dazu auch: Maria spinnt im Tempel, als Tempeljungfrau (Eibner 1986).

8) Vgl. dazu auch die ebenfalls nur fragmentiert überlieferte Bronzestatuette eines solchen Kämpfers, geschmückt mit Oberarmringen, aus Hallstatt, Grab 585 (A) (Kruta 1993, 201 mit Abb. 170).

9) Solche Figuren wie Frauen mit erhobenen Armen, Reiter sowie auch Musikanten, vor allem Leierspieler (Abb. 6) finden sich zum Teil auch in Graphitmalerei auf Kegelhalsgefäßen bzw. im Inneren einer Schale vor allem in reicher ausgestatteten Gräbern der Kalenderbergkultur (Eibner 1997, 143/Abb. 53).

10) Vgl. zur senkrecht gespaltenen Kontrastbemalung dieser Körper die Beinschienen aus Silber von der Mogilanska Mogila in Varca (BG), wo die rechte Wange des Frauenkopfes goldene Streifen zeigt, während die linke silberfarben ist (Venedikov - Gerassimov 1973, 86 f. u. 349 mit Abb./231 u. 232).

11) Vergleiche dazu den Amazonen-Mythos: Preinfalk 2003, 85/Sp. 2 mit Anm. 528; Teržan - Hellmuth - Heimann 2011, bes. 253 ff./I. Prolog.

12) Das verbreiterte Becken der eindeutig männlichen Figuren aus Langenlebarn, Tumulus 3 (A), zeigt sich m. E. im Umriss allerdings schon von anderer Gestalt als das rockartige Gewand der Gemeinlebarner Frauenstatuetten, das eindeutig zipfelartig - dreieckig wie ein Rock - vom Körper absteht (vgl. Preinfalk 2003, 85/Sp. 1 mit 85/Sp. 2 ; vgl. dazu Dungel - Szombathy 1890, 58-Bekleidung u. 57 mit Taf. II: Fig. 2 u. 4).

13) Es sind zwei Gefäße erhalten, ein beckenförmiges und eine Rippenziste, wobei für beide der Birkenteer als Klebemittel verwendet wurde (Abb. 5: 1) (Dungel - Szombathy 1890, 59 mit Fig. 29 u. Taf. II: Fig. 5-6; Preinfalk 2003, 85 mit Anm. 523-524 u. 92; zum Birkenteer vgl. Persy [Eibner] 1967). 
14) Ähnlich den Keramikgefäßen mit den seitlich nach oben gestreckten Armen von Nové Košariská (SK) und Marz (A) sowie den Bronzefiguren, die die Bronzekline von Hochdorf (D) mit ihren erhobenen Armen abstützen (Abb. 5: 2).

15) Vgl. dazu Dungel - Szombathy 1890, 56 ff./b) Gefäße: 17. Drei rothe Urnen - 57: „Meiner Meinung nach besassen diese Gefässe auch noch plastischen Schmuck. Es wurden in der unmittelbaren Nähe derselben kleine Bronzevögelchen und thönerne Figürchen gefunden, welche mit den Urnen höchst wahrscheinlich im Zusammenhange stehen.“ (vgl. hier Abb. 4: 3); bes. 60-62 mit Fig. 39-60: „Bezüglich der Plätze, an welchen diese Bronze- und Thonfigürchen ihre Aufstellung gefunden haben mögen, sind wir durchwegs auf unsere Schlüsse angewiesen, da keine directen Beobachtungen vorliegen.“; 61: „... finde ich in gewissen Abständen die Spuren von kleinen Löchern, welche bei der ersten Zusammenstellung als zufällige Beschädigungen betrachtet und verklebt worden waren.“ . . „Dieses Zusammentreffen ... berechtigt wohl zu der Annahme, dass die Thonfigürchen den Umfang der Urne geziert haben ... .“; 62: „Ich habe wohl auch sechs schwarze Urnen ... mit geeigneten Löchern im Mundsaum gefunden, aber die Stellen für die Anbringung der Thonfigürchen kann ich dort nicht so genau nachweisen wie bei den rothen Urnen."

16) Bei einigen Fröger Bleifiguren (A) ist an eine Anbringung auf einem hölzernen Situlendeckel bzw. auf einem hölzernen Leichenbrandbehälter gedacht worden (Preinfalk 2003, 85 mit Anm. 525). - Vgl. dazu auch die Bronzemaske und -hände aus dem Kröllkogel von Kleinklein (A), die ursprünglich auf einem hölzernen Gegenstand befestigt gewesen waren - wohl auf Holz montierte Elemente einer Büste -, die möglicherweise den Toten darstellte, ähnlich den Büsten in etruskischen Gräbern ( $\operatorname{Egg}$ 2013, 466 f. mit Abb. 208: 1-2-Vergleich der Büsten auf der Ziste XIII mit der Bronzemaske und den Händen des Kröllkogels).

17) „..., dass es sich um Menschen- und verschiedene Thiergestalten handelt, welche mittelst eines schwarzen beim Erhitzen wohlriechenden Harzes auf einer schiefen Thongefässfläche aufgeklebt waren.“ „Die Stellung der an den Beinen noch erhaltenen kleinen Harzkuchen zeigt, dass die Thonfigürchen alle mit dem Rücken gegen eine aufsteigende Fläche, wie es etwa der Hals unserer grossen Urnen ist, gekehrt waren." (Dungel Szombathy 1890, 57 u. 59 mit 58/Fig. 31) (vgl. dazu Abb. 4: 3). - Zur Teerverwendung für Flickarbeiten vgl. Dungel - Szombathy 1890, 59- aus Teer geformte Arme wurden an Stelle der abgebrochenen keramischen angeklebt.

18) Vgl. dazu den nackten Mann mit Beil und Lanze auf der Situla Sanzeno (I) (vgl. hier Anm. 2) sowie die auf den Situlen Este-Benvenuti und Montebelluna (beide: I) dargestellten Beile - liegend unter dem Regal mit den aufgehängten Trankbehältern bzw. aufgehängt über dem Bett mit dem Liebespaar (Eibner 2016b, 74 mit Taf. 8: a u. f) - und das Beil und die Bratspieße, die der letzte Mann im Prozessionszug auf der Situla Certosa mit sich trägt (Abb. 7). 


\section{Literaturverzeichnis}

Aigner-Foresti, L. 2010: Etrurien und die Gebiete nördlich der Alpen in der Villanova-Zeit: Kulturelle Beziehungen und historischer Hintergrund. In: Gediga, B. - Piotrowski, W. (Hrsg.) 2010, 127$143 / 145$.

Arbeitskreis Falkenberg (Hrsg.) o. J. [2015]: Die Hallstattfürsten von Falkenberg. Die Region Murtal erforscht ihre Geschichte. 5 Jahre Arbeitskreis Falkenberg. Entdecken - Ergraben - Erforschen - Bewahren. Judenburg.

Belanová, T. - Čambal, R. - Stegmann-Rajtár, S. 2007: Die Weberin von Nové Košariská - Die Webstuhlbefunde in der Siedlung von Nové Košariská im Vergleich mit ähnlichen Fundplätzen des östlichen Hallstattkulturkreises. In: Blečić, M. - Črešnar, M. - Hänsel, B. - Hellmuth, A. - Kaiser, E. - NebelsickMetzner, C. (Hrsg.): Scripta praehistorica in honorem Biba Teržan (zum 60. Geburtstag). Situla 44 (Ljubljana), 419-434.

Berg, F. 1962: Das Flachgräberfeld der Hallstattkultur von Maiersch. Veröffentlichungen der Österreichischen Arbeitsgemeinschaft für Ur- und Frühgeschichte IV, Wien.

Biel, J. 1985: Der Keltenfürst von Hochdorf. Stuttgart.

Chytráček, M. - Chvojka, O. - Egg, M. - John, J. - Kyselý, R. - Michálek, J. - Ritter, St. - Stránská, P. 2015: $\mathrm{Zu}$ einem Fürstengrab aus der Späthallstattzeit mit zweirädrigem Wagen und Bronzegefäßen bei Rovná (okr. Strakonice) in Südböhmen, Archäologisches Korrespondenzblatt 45, 71-89.

Dungel, A. - Szombathy, J. 1890: Die Tumuli von Gemeinlebarn. Ausgegraben von Dr. Adalbert Dungel, beschrieben von Josef Szombathy, Mittheilungen der Prähistorischen Commission der kaiserlichen Akademie der Wissenschaften I, No 2, Wien, 49-77.

Egg, M. 1996: Das hallstattzeitliche Fürstengrab von Strettweg bei Judenburg in der Obersteiermark. Monographien des Römisch-Germanischen Zentralmuseums Mainz 37, Mainz.

Egg, M. 2013: Zur figuralen Kunst von Kleinklein. In: Egg, M. - Kramer, D. (Hrsg.): Die hallstattzeitlichen Fürstengräber von Kleinklein in der Steiermark: der Kröllkogel. Monographien des Römisch-Germanischen Zentralmuseums Mainz 110, Mainz, 447-472.
Eibner, A. 1974: Zum Befund einer hallstattzeitlichen Webgrube aus Stillfried. Forschungen in Stillfried $1=$ Veröffentlichungen der Österreichischen Arbeitsgemeinschaft für Ur- und Frühgeschichte VI, Wien, 76-84.

Eibner-Persy, A. 1980: Hallstattzeitliche Grabhügel von Sopron (Ödenburg). Die Funde der Grabungen 1890-92 in der Prähistorischen Abteilung des Naturhistorischen Museums in Wien und im Burgenländischen Landesmuseum in Eisenstadt. Wissenschaftliche Arbeiten aus dem Burgenland 62, Eisenstadt (= Gedruckte Dissertation, Wien 1966).

Eibner, A. 1986: Die Frau mit der Spindel. Zum Aussagewert einer archäologischen Quelle. In: Hallstatt-Kolloquium Veszprém 1984. Mitteilungen des Archäologischen Institutes der Ungarischen Akademie der Wissenschaften Beiheft 3, Budapest, 39-48.

Eibner, A. 1993: Zur Lesbarkeit der Bildsymbolik im Osthallstattkreis, Thraco-Dacica XIV, 101-116.

Eibner, A. 1997: Die „Große Göttin“ und andere Vorstellungsinhalte der östlichen Hallstattkultur. In: Nebelsick, L. - Eibner, A. - Lauermann, E. - Neugebauer, J.-W. (Hrsg.) 1997, 129-145.

Eibner, A. 1999: Der Aulosbläser von Százhalombatta und sein kulturgeschichtliches Umfeld. Versuch einer Interpretation. In: Jerem, E. - Poroszlai, I. (Hrsg.): Archaeology of the Bronze and Iron Age. Proceedings of the International Archaeological Conference Százhalombatta, 3-7 October 1996. Archaeolingua 9, Budapest, 37-52.

Eibner, A. 2000/2001: Die Stellung der Frau in der Hallstattkultur anhand bildlicher Zeugnisse, Mitteilungen der Anthropologischen Gesellschaft in Wien CXXX/CXXXI, 107-136.

Eibner, A. 2004: Die Bedeutung der Jagd im Leben der eisenzeitlichen Gesellschaft - dargestellt anhand der Bildüberlieferungen. In: Heftner, H. Tomaschitz, K. (Hrsg.): Ad fontes! Festschrift für Gerhard Dobesch zum 65. Geburtstag am 15. September 2004. Wien, 621-644.

Eibner, A. 2005: Wie wertvoll ist eine Frau?! Gedanken zum Stellenwert des Spinnens und Webens in der Gesellschaft. In: Feldinger, E.M. (Hrsg.): Scherben bringen Glück. Festschrift Fritz Moosleitner zum 70. Geburtstag. Salzburg, 31-38. 
Eibner, A. 2012: Die Situla von Kuffern. Zu Ritualen und identitätsstiftenden Handlungskomplexen in der Situlenkunst. In: Danek, G. - Hellerschmid, I. (Hrsg.): Rituale. Identitätsstiftende Handlungskomplexe. 2. Tagung des Zentrums Archäologie und Altertumswissenschaften an der Österreichischen Akademie der Wissenschaften, Wien 2.-3. November 2009. Origines, Schriften des Zentrums Archäologie und Altertumswissenschaften 2, Österreichische Akademie der Wissenschaften phil.-hist. Klasse Denkschriften 437, Wien, 47-69.

Eibner, A. 2014: Arbeitsdarstellungen in der Situlenkunst und in benachbarten Gebieten, Mitteilungen der Anthropologischen Gesellschaft in Wien CXLIV, 35-60.

Eibner, A. 2015: Feste und Rituale in der Situlenkunst, Mitteilungen der Anthropologischen Gesellschaft in Wien CXLV, 55-84.

Eibner, A. 2016a: Kulturelle Einflussnahme aus Etrurien auf die benachbarten Kulturräume der europäischen Eisenzeit. In: Gediga, B. - Grosman, A. - Piotrowski, W. (Hrsg.) 2016, 147-193/195.

Eibner, A. 2016b: Überlegungen zur Einrichtung eines eisenzeitlichen Hauses der Oberschicht - anhand von archäologischen und bildlichen Quellen, Mitteilungen der Anthropologischen Gesellschaft in Wien CXLVI, 59-87.

Eibner, C. 2012: Hallstattzeitliche Sagen und Mythen. In: Anreiter, P. - Bánffy, E. - Bartosiewicz, L. - Meid, W. - Metzner-Nebelsick, C. (Eds.): Archaeological, Cultural and Linguistic Heritage. Festschrift for Erzsébet Jerem in Honour of her $70^{\text {th }}$ Birthday. Archaeolingua 25, Budapest, 195-205.

Eibner, C. 2016: Siedlungen entlang der Bernsteinstraße und ihre kulturelle Veränderung in der Eisenzeit. In: Gediga, B. - Grossman, A. - Piotrowski, W. (Hrsg.) 2016, 15-24.

Gediga, B. 2010: Schlesien - regionale Provinz der Hallstattkultur. In: Gediga, B. - Piotrowski, W. (Hrsg.) 2010, 187-216/218.

Gediga, B. 2011: Neue Forschungen zu den früheisenzeitlichen Kulturen in Südwestpolen, Acta Archaeologica Carpathica XLVI, 83-116.

Gediga, B. - Piotrowski, W. (Hrsg.) 2010: Rolle der wichtigen Kulturzentren in der Gestaltung des Kulturbildes Mitteleuropas in den frühen Perioden der Eisenzeit. Biskupiner Archäologische Arbeiten 8
/ Polnische Akademie der Wissenschaften - Abteilung Wrocław, Arbeiten der Archäologischen Kommission 18, Biskupin-Wrocław.

Gediga, B. - Grossman, A. - Piotrowski, W. (Hrsg.) 2016: Europa zwischen 8 Jhd. v. Chr. Geb. bis 1 Jhd. u. Zeit. Biskupiner Archäologische Arbeiten 11 / Polnische Akademie der Wissenschaften - Abteilung Wrocław, Arbeiten der Archäologischen Kommission 21, Biskupin-Wrocław.

Gleirscher, P. 2009: Sopron - Nové Košariská - Frög. $\mathrm{Zu}$ den Bildgeschichten der Kalenderberg-Kultur, Prähistorische Zeitschrift 84, 202-223.

Gleirscher, P. - Nothdurfter, H. - Schubert, E. 2002: Das Runger Egg. Untersuchungen an einem eisenzeitlichen Brandopferplatz bei Seis am Schlern in Südtirol. Römisch-Germanische Forschungen 61, Mainz.

Gömốri, J. 2002: Grab der Osthallstattkultur mit Wagengefäß aus Fertőendréd (Kom. Sopron, Ungarn). In: URL www.ag-eisenzeit.de/2002 (Sopron), sopron_abstracts.pdf, 12-13.

Kašuba, M. 2011: Erscheinungsformen hallstattzeitlicher Stammesfeste im Nordpontikum am Beispiel der Malteserkreuzdarstellungen (2. Hälfte 8.-7. Jh. v.Chr.). In: Sava, E. - Govedarica, B. - Hänsel, B. (Hrsg.) 2011, 237-252.

Kromer, K. 1958: Gemeinlebarn, Hügel 1. Inventaria Archaeologica, Österreich Heft 2, Bonn.

Kruta, V. 1993: Die Anfänge Europas von 6000 bis 500 v. Chr. Universum der Kunst 38, München.

Łaciak, D. - Markiewicz, M. 2013: Painted ceramics of Hallstatt period cemetery at Domasław, site 10/11/12, distr. Wrocław. In: Kolendy, J. Mierzwiński, A. - Moździoch, S. - Źygadto, L. (Red.): $\mathrm{Z}$ badań nad kulturą społeczeństw pradziejowych i wczesnośredniowiecznych. Księga Jubileuszowa dedykowana Profesorowi Bogusławowi Gedidze, w osiemdziesiątą rocznicę urodzin przez przyjaciól, kolegów i uczniów. Wrocław, 527-540.

Lucke, W. - Frey, O.-H. 1962: Die Situla in Providence (Rhode Island). Ein Beitrag zur Situlenkunst des Osthallstattkreises. Römisch-Germanische Forschungen 26, Berlin.

Nebelsick, L. 1997: Die Kalenderberggruppe der Hallstattzeit am Nordostalpenrand. In: Nebelsick, L. - Eibner, A. - Lauermann, E. - Neugebauer, J.-W. (Hrsg.) 1997, 9-128. 
Nebelsick, L. - Eibner, A. - Lauermann, E. - Neugebauer, J.-W. (Hrsg.) 1997: Die Hallstattkultur im Osten Österreichs. Wissenschaftliche Schriftenreihe Niederösterreich 106/107/108/109, St. Pölten.

Neugebauer, J.-W. 1988: Neuere Forschungsergebnisse auf dem Gebiet der Hallstattkultur in Nordösterreich. Archäologie Alpen Adria 1 (Festschrift 100 Jahre Ausgrabungen in Frög), Klagenfurt, 85-107.

Neugebauer, J.-W. 1997: Beiträge zur Erschließung der Hallstattkultur im Zentralraum Niederösterreichs. In: Nebelsick, L. - Eibner, A. - Lauermann, E. - Neugebauer, J.-W. (Hrsg.) 1997, 165-190.

Persy [Eibner], A. 1967: Bemerkungen zum „Harz“Überzug auf hallstattzeitlichen Gefäßen aus Ödenburg, Archaeologia Austriaca 41, 22-24.

Preinfalk, F. 2003: Die hallstattzeitlichen Hügelgräber von Langenlebarn, Niederösterreich. Fundberichte aus Österreich, Materialhefte Reihe A, Heft 12, Wien.

Sauter, F. - Graf, A. - Hametner, Ch. - Fröhlich, J. Neugebauer, J.-W. - Preinfalk, F. 2002: Studies in Organic Archaeometry IV: Analysis of an organic agglutinant used to fix Iron-age clay figurines to their base. ARKIVOC Vol. 2000, Part (I), no. 6, 35-39. Full Text: http://www.arkat-usa.org $\Rightarrow$ Volume $2002 \Rightarrow$ Part (I) $\Rightarrow$ no 6.

Sava, E. - Govedarica, B. - Hänsel, B. (Hrsg.) 2011:

Der Schwarzmeerraum vom Äneolithikum bis in die Früheisenzeit (5000-500 v. Chr.). Band 2: Globale Entwicklung versus Lokalgeschehen. Internationale Fachtagung von Humboldtianern für Humboldtianer im Humboldt-Kolleg in Chişinău, Moldavien (4.-8. Oktober 2010). Prähistorische Archäologie in Südosteuropa 27, Rahden/Westf. Studeniková, E. 1995: Hallstattzeitlicher Tumulus II in Janíky, Bez. Dunajská streda. Vorläufige Grabungsergebnisse, Zborník Slovenského národného múzea LXXXIX, Archeológia 5, 49-75.

Teržan, B. - Hellmuth, A. - Heimann, F. 2011: Amazonenmythos im Spiegel der eisenzeitlichen Grabfunde zwischen Pontus und Karpatenbecken. In: Sava, E. - Govedarica, B. - Hänsel, B. (Hrsg.) 2011, 253-272.

Tiefengraber, S. u. G. o. J. [2015]: Die „Fürstengräber“ von Strettweg und Waltersdorf. Eine archäologische Suche nach den Spuren längst vergangener Zeiten. In: Arbeitskreis Falkenberg (Hrsg.) o. J. [2015], 25-60.

Totschnig, R. o. J. [2015]: Archäologisch-Geophysikalische Prospektion in Judenburg-Strettweg/ Waltersdorf. In: Arbeitskreis Falkenberg (Hrsg.) o. J. [2015], 16-23.

Venedikov, I. - Gerassimov, T. 1973: Thrakische Kunst. Wien-München.

Woytowitsch, E. 1978: Die Wagen der Bronze- und frühen Eisenzeit in Italien. Prähistorische Bronzefunde XVII/1, München. 


\section{Kultwagen mit Figuren aus Keramik im Osthallstattkreis?}

Im bronzenen Kultwagen von Strettweg besitzen wir für den Osthallstattkreis einen gefäßtragenden Wagen, wie er sonst im nördlichen und mittleren Italien zu finden ist, wobei hier die in Durchbruchstechnik hergestellte zyprische Szenerie durch Kleinplastiken ersetzt wird. Das Prinzip der zwei Ebenen dieser Kesselwagen, einer figurierten unteren und einer oberen zur Aufnahme des Gefäßes, ist hier durch den spiegelbildlich beidseits die Statue begleitenden Prozessionszug sowie durch die große, nackte weibliche Statuette in der Mitte des Wagens gegeben, die ein mit den Händen gestütztes Gefäß am Kopf trägt. Nachdem es in der Kalenderbergkultur Figürchen aus Bronze bzw. aus gebranntem Ton (= Keramik) gibt, stellt sich die Frage, wo diese montiert gewesen waren, nachdem sich an deren Beinen Nietlöcher bzw. Teerklumpen zum Einsetzen in eine Unterlage gefunden haben.

Da wir so gut wie keine Bronzegefäße in den Gräbern der Kalenderbergkultur besitzen, dürfen wir hier m. E. dieselben in Keramik umgesetzt erwarten.

So lässt sich als ein Beispiel für die Umsetzung eines Bronzeobjektes - eines gefäßtragenden Bronzewagens - in Keramik ein vierrädriger Tonwagen mit einem Kalenderberggefäß als Aufsatz aus Fertőendréd, Kom. Sopron (H), anführen, dem ein gleichartiger tönerner Wagen mit einem aufgesetzten bemalten Gefäß aus Domasław, Kr. Wrocław (PL), in der Randzone, der nordöstlichen Provinz der Hallstattkultur gelegen, an die Seite zu stellen ist.

Uns stehen für eine solche Umsetzung zwei keramische Fundensembles aus reich ausgestatteten Gräbern der Kalenderbergkultur zur Verfügung: Das eine mit männlichen Figuren ausgestattet, stammt aus Langenlebarn (A), das andere mit weiblichen Statuetten aus Gemeinlebarn (A), wobei sich die weiteren Plastiken gleicherweise aus Pferden, Reitern und auch einem Hirsch zusammensetzen. M. E. liegen daher mehr oder weniger die gleichen Menschentypen und Tiere vor, die auch auf dem Kultwagen zu finden sind - nur in Keramik ausgeführt.

Vermutlich wurden daher diese Kultwagen - wie Strettweg (A) mit Figurenbesatz - in Ton nachgebildet bzw. auf eine organische Unterlage wie z. B. auf einen aus Holz hergestellten Wagen eingeklebt.

In diesem Bildmaterial lässt sich wohl eine Schilderung von Kulthandlungen bzw. Prozessionen oder Mythen erkennen (Abb. 1; 3: 1), die sich m. E. auch in den Darstellungen der Situlenkunst (Abb. 7; 5: 3) sowie auf den Zisten aus dem Kröllkogel von Kleinklein (A) (Abb. 2), aber auch in den figuralen Darstellungen der Kalenderbergkultur (Abb. 3: 2; 4: 1-3; 5 : 1 u. $4 ; 6$ ) wiederfinden - nur jeweils in einem anderen Kunstmittel ausgedrückt.

\section{Prof. Dr. Alexandrine Eibner}

- Alszeile 118/10/4, A-1170 Wien, Österreich

alexandrine.eibner@gmx.at 